\title{
Warum suchen Biotechnologieunternehmen strategische Allianzen? Eine experimentelle Untersuchung
}

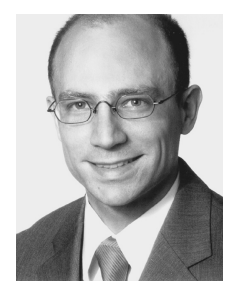

\section{Holger Patzelt*}

\section{Überblick}

- Dieser Beitrag untersucht wie sich die Ressourcensituation eines jungen Unternehmens auf die Motivation zur Allianzsuche der Manager auswirkt.

- Grundlage des Beitrags bildet ein Conjoint-Experiment mit 52 Top-Managern deutscher Biotechnologieunternehmen.

- Eine Analyse von 1664 Allianzentscheidungen der Manager zeigt, dass fehlende finanzielle Ressourcen die Haupttriebkraft der Allianzsuche sind.

- Zudem zeigt sich, dass sowohl fehlende als auch vorhandene Ressourcen eine Motivation zur Bildung einer neuen Allianz darstellen können.

- Die Ergebnisse der Studie erweitern die wissenschaftliche Literatur zu strategischen Allianzen.

- Implikationen für die Managementpraxis werden diskutiert.

Keywords Biotechnology $\cdot$ alliance $\cdot$ resources $\cdot$ decision making

Eingegangen: 30. November 2006

JEL: M13

Dr. rer. nat. Dr. rer. pol. Holger Patzelt ( $\square$ )

Senior Research Fellow, Max-Planck-Institut für Ökonomik, Kahlaische Str. 10, 07745 Jena, Tel.: 03641686 726, Fax: 03641686 710, patzelt@econ.mpg.de, URL: http://www.econ.mpg.de 


\section{A. Einleitung}

Strategische Allianzen sind ein verbreitetes Phänomen in der Biotechnologiebranche. Europaweit wurden allein im Jahre 2001 über 500 dieser freiwilligen Firmenkooperationen beobachtet (Ernst \& Young, 2002). In Deutschland belief sich die Zahl im Jahr 2004 auf 147, eine Steigerung von 40 \% gegenüber dem Vorjahr (Ernst \& Young, 2005, S. 80). Obwohl in der Literatur Motive für die Allianzbildung zwischen Technologieunternehmen wie die Teilung von Entwicklungsrisiken (Hamel et al., 1989), die Gewinnung von Marktmacht (Hagedoorn, 1993), oder eine Erhöhung der Legitimität und Visibilität des Unternehmens (Baum, Oliver, 1991) beschrieben werden, haben sich Studien bisher auf eine ex post-Perspektive beschränkt, d. h. bestehende Allianzen untersucht ohne zu berücksichtigen, dass viele Firmen vergeblich nach Allianzpartnern suchen. In dieser Arbeit sollen diese Limitationen adressiert und aus einer ex ante-Perspektive die Triebkräfte der Allianzsuche von Biotechnologiefirmen analysiert werden.

Ausgehend von dem ressourcenorientierten Ansatz wird postuliert, dass die Ressourcensituation eines Unternehmens sowie die Ressourcenanforderungen bzw. -akquisitionsmöglichkeiten der Umwelt ausschlaggebend für die Bildung von Allianzen sind (Gomes-Casseres, 1996). Unter Ressourcen werden alle tangiblen und intangiblen Güter einer Firma verstanden, die dazu beitragen, Produkte und Dienstleistungen zu entwickeln, zu produzieren und zu vermarkten (Barney, 1991; zu Knyphausen, 1993). Es ist erstaunlich, dass bislang noch keine Arbeit untersucht hat, ob und welche direkten Auswirkungen diese Faktoren auf die Allianzentscheidungen von Managern haben, insbesondere welche relative Bedeutung diese Faktoren haben. In diesem Artikel wird versucht, diese Forschungslücken zu füllen. Hierzu werden 1664 Entscheidungen aus einem Conjoint-Experiment mit 52 Top-Managern der deutschen Biotechnologiebranche untersucht. Dadurch werden folgende Forschungsbeiträge zur Literatur über strategische Allianzen gemacht.

Zum ersten liefert die Arbeit einen Beweis dafür, dass die Ressourcenausstattung und die Umwelt eines Unternehmens wichtige Vorläufer der Allianzentscheidung von Managern sind. Existierende Arbeiten bieten wenig Einblick, wie Heterogenitäten in der Ressourcenausstattung, die entscheidend für die Entscheidungsfindung von Managern sind, deren Allianzsuche motivieren, insbesondere welche relative Bedeutung Motive der Allianzsuche haben. In der vorliegenden Arbeit wird für den speziellen Fall der Produktentwicklungsallianzen junger Biotechnologiefirmen die Einflussstärke der Motive ermittelt. Schließlich beschreibt die wissenschaftliche Literatur zwar, dass prinzipiell sowohl fehlende als auch vorhandene Ressourcen Triebkraft einer Allianzbildung darstellen können (Das, Teng, 2000), fundierte empirische Analysen dieser theoretischen Argumentation fehlen aber. Die vorliegende Arbeit zeigt, dass zwar für die meisten Manager junger Biotechnologiefirmen ein Ressourcendefizit die Hauptmotivation zur Allianzbildung darstellt, ein nicht unwesentlicher Anteil der Manager jedoch eher durch vorhandene als fehlende Ressourcen zur Allianzsuche motiviert wird.

Im Folgenden werden zunächst die Theorie und die Hypothesen formuliert. Im Anschluss werden die Stichprobenauswahl, die Eigenschaften der Stichprobe und die Forschungsmethode beschrieben. Es folgen eine Darstellung der Ergebnisse sowie eine abschließende Diskussion. 


\section{B. Theoriebildung}

Das Bestreben, wertvolle Ressourcen zu akquirieren, ist einer der theoretischen Hauptgründe für die Bildung von Allianzen zwischen Unternehmen. Im Rahmen dieser Studie wird eine strategische Allianz als jede Art freiwilliger Firmenkooperation mit dem Ziel, Wettbewerbsvorteile für die Allianzpartner zu erzielen, verstanden (Das, Teng, 2000, S. 33). Diese Definition beinhaltet eine Vielzahl verschiedener vertraglicher Regelungen wie z. B. Lizenzierungen, Technologieaustausch, Joint Ventures und Minderheitsbeteiligungen (Gulati, 1995).

Junge Technologieunternehmen sind durch hohe Ressourcenbedürfnisse und minimale Ressourcenausstattung gekennzeichnet, weshalb die Akquisition von Ressourcen eine der Hauptaufgaben ihrer Gründer und Manager darstellt (Brush et al., 2001). Diese Aufgabe ist besonders in forschungsintensiven Industriezweigen wie der Biotechnologie herausfordernd, da die Produktentwicklung ein extrem teurer und zeitintensiver Prozess ist (Herstatt, Müller, 2002). Andererseits haben junge Firmen oftmals ein höheres Innovationspotential als etablierte Unternehmen, da ihr spezielles „Klima“ und ihre unternehmerische Kultur essentiell für die Entwicklung radikal innovativer Produkte sind (Schweizer, 2005). Große Pharmafirmen setzen deshalb Allianzen mit jungen Biotechnologieunternehmen ein um sich den Zugriff auf viel versprechende Produktkandidaten sowie das zu deren Entwicklung nötige Wissen zu verschaffen (Rothaermel, Deeds, 2004).

Aus Sicht eines jungen Biotechnologieunternehmens jedoch wird die Entscheidung zur Allianzsuche von Defiziten in der Ressourcenausstattung sowie von den Ressourcenanforderungen der Umwelt bzw. der Möglichkeit, Ressourcen über alternative Quellen aus der Umwelt zu beziehen, abhängen.

\section{Einfluss der Ressourcenausstattung des Unternehmens}

In Hochtechnologieindustrien wie der Biotechnologie fehlen jungen Firmen oftmals eine Vielzahl von Ressourcen zur weiteren Produktentwicklung, was Manager veranlasst, Zugang zu diesen Ressourcen über strategische Allianzen zu suchen (Chi, 1994; Hagedoorn, 1993). Studien haben gezeigt, dass wichtige Ressourcen wie z. B. Wissen und Produktions- und Vermarktungskapazitäten über strategische Allianzen von Unternehmen akquiriert werden können (Audretsch, Feldman, 2003). Auch nach Abschluss der Produktentwicklung verfügen die Firmen oftmals nicht über die Ressourcen, die für eine erfolgreiche Markteinführung notwendig sind, was eine Suche nach Vermarktungsallianzen mit etablierten Firmen zur Folge hat (Audretsch, Feldman, 2003; Hagedoorn, 1993). Da die meisten biopharmazeutischen Unternehmen der jungen deutschen Branche - die den Untersuchungsgegenstand dieser Arbeit bildet - jedoch noch weit von der Markteinführung ihrer Produkte entfernt sind (bis zum Jahr 2005 hatte nur ein deutsches Biotech-Unternehmen ein Medikament zur Marktreife gebracht, Ernst \& Young, 2005), wird im Folgenden ausschließlich auf Produktentwicklungsallianzen abgestellt.

Die vorhandene Literatur nennt eine Reihe von Ressourcen, die zum Erfolg junger Biotechnologieunternehmen beitragen. Erstens sind finanzielle Ressourcen entscheidend, da sie fungibel sind und es dem Unternehmen erlauben, andere Ressourcen über die Fak- 
tormärkte zu beziehen (Dollinger, 1995). Insbesondere in der Biotechnologiebranche, wo Produktentwicklung mit hoher Unsicherheit und langen Entwicklungszyklen verbunden ist, sind große Mengen an finanziellen Ressourcen erforderlich. Die Entwicklung von Biopharmazeutika z. B. verschlingt mehrere Hundert Millionen $€$ und benötigt mehr als 10 Jahre (DiMasi et al., 2003). Allianzen mit anderen Unternehmen sind für Biotechnologiefirmen eine Möglichkeit zur Akquisition finanzieller Ressourcen, da sie häufig Finanzierung von Forschung, Equity-Investitionen und andere Arten direkter Finanzierung beinhalten (DeCarolis, Deeds, 1999). So zeigen Coombs et al. (2006), dass sowohl nationale als auch internationale Allianzen von Biotechnologiefirmen zu direkten Zuflüssen finanzieller Mittel führen und dass die akquirierte Menge sowohl von firmen- als auch von standortspezifischen Faktoren sowie von der Firmengröße abhängt. Ein prominentes Beispiel für die direkte Finanzierungsfunktion von Allianzen in der deutschen Biotechnologiebranche ist die Firma Mikromet, die in Jahr 200114 Millionen US-Dollar Vorabzahlung für eine Allianz von ihrem Partner Enzon, und im Jahre 200410 Millionen USDollar Vorabzahlung von Serono akquirieren konnte (Wess, 2002a). Auch für Firmen wie Ingenium oder Epidauros (Wess, 2002b; Zoltobrocki, 2005) sowie viele andere sind Allianzen ein essentielles Instrument zur Generierung eines Cash-Flow.

Zweitens sind strategische Allianzen für Biotechnologiefirmen eine Möglichkeit, ihre Produktpipeline zu erweitern (Deeds et al., 1997, 1998). Produktkandidaten sich wichtige Ressourcen für Biotechnologiefirmen und tragen substanziell zum Unternehmenswert bei (Rothaermel, Deeds, 2004). Manager von Biotechnologieunternehmen müssen versuchen, eine ausgewogene Produktpipeline aufzubauen, die Produkte in späten Entwicklungsstadien enthält, aber auch solche in frühen Stadien, die Ausfälle in späten Stadien kompensieren können. Sowohl fehlende frühe als auch fehlende späte Produktkandidaten werden Manager dazu veranlassen, nach einer Alternative zur Erhöhung der Entwicklungsrate zu suchen, wie es im Rahmen einer strategischen Allianz möglich ist (Deeds, Hill, 1996).

Drittens sind Patente eine wichtige Ressource für Biotechnologiefirmen (Ernst, Omland, 2003). Es ist wichtig zu betonen, dass, obwohl Produktkandidaten in der Regel durch Patente geschützt sind, die Zahl der Patente diejenige der Produktkandidaten meist weit übersteigt. Patentiert werden von Biotechnologieunternehmen auch Prozesse und grundlegende Verfahren und Erkenntnisse, die erst - und nicht in jedem Fall - zukünftig für die Produktentwicklung von Nutzen sein können. Die Zahl der Patente einer Biotechnologiefirma ist ein wichtiger Indikator für ihre technologische Stärke und Innovationskraft (Acs, Audretsch, 1989). Da im Rahmen strategischer Allianzen die Partner oftmals gemeinsame Patente entwickeln, werden Manager versuchen, einer schwachen Patentsituation ihrer Firma durch verstärkte Allianzsuche zu begegnen.

Viertens wird in der Literatur die Wichtigkeit von Humanressourcen, insbesondere eines Teams exzellenter Wissenschaftler, für die Produktentwicklung in Biotechnologieunternehmen erwähnt (Deeds et al., 1999). Wissenschaftler generieren Wissen und Expertise, die essentielle Ressourcen darstellen (DeCarolis, Deeds, 1999). Dabei kommt in Technologiefirmen insbesondere einer kleinen Gruppe von Schlüsselerfindern eine tragende Rolle zu (Ernst et al., 1999). Falls ein Biotechnologieunternehmen nicht über hochqualifizierte Wissenschaftler verfügt, werden die Manager verstärkt auf Wissen anderer Organisationen zugreifen müssen und daher Allianzen suchen. 
Schließlich stellt das existierende Kontaktnetzwerk für eine Biotechnologiefirma eine wertvolle Ressource dar. Das Kontaktnetzwerk einer Firma ist die Summe aller Allianzen und informellen Kontakte zu anderen Firmen, Universitäten, Forschungsinstituten und Investoren (Powell et al., 1996). Netzwerke sind eng an die Firma gebunden und damit ein wichtiger Wettbewerbsvorteil (Das, Teng, 2000). Sie sind eine entscheidende Quelle für neues Wissen (Witt, Rosenkranz, 2002) und erhöhen die strategische Flexibilität und technologische Kompetenz (Zucker et al., 2002). Die Knüpfung einer neuen Allianz mit einer anderen Firma bietet oftmals den Zugang zum Netzwerk des Partners (Gulati, 1995).

\section{Einfluss der Umwelt des Unternehmens}

Die Umwelt von Biotechnologieunternehmen beeinflusst aus einer ressourcenorientierten Perspektive das Verhalten von Managern auf zwei Arten: zum einen stellt die Umwelt Anforderungen an die Ressourcenausstattung des Unternehmens, und zum anderen bieten sich für den Manager Möglichkeiten, Ressourcen aus der Umwelt zu akquirieren (GomesCasseres, 1996). Erstes wird im Rahmen dieser Studie durch die Konkurrenzsituation des Unternehmens abgebildet (Eisenhardt, Schoonhoven, 1996), wohingegen die Ressourcenakquisitionsmöglichkeit durch die Situation an den Kapitalmärkten beschrieben wird (Fildes, 1990).

In einem Umfeld mit starkem Konkurrenzdruck sind Technologieunternehmen gezwungen, möglichst schnell mit ihren innovativen Produkten in den Markt einzutreten um maximalen Erfolg zu erzielen. In diesen „verwundbaren“ strategischen Situationen besteht für Manager eine Notwendigkeit, Allianzen mit anderen Unternehmen zu suchen um die Ressourcensituation ihres Unternehmens zu stärken und die Geschwindigkeit der Produktentwicklung zu erhöhen (Eisenhardt, Schoonhoven, 1996).

Da die meisten jungen Biotechnologieunternehmen noch keine substantiellen Umsätze erzielen, hängt ihre Entwicklung essentiell von der Kapitalinfusion durch Investoren ab. Deshalb ist die Akquisition von Kapital eine der Hauptaufgaben ihrer Manager (Fildes, 1990). Die Möglichkeit der Kapitalakquisition ist jedoch im Zeitablauf nicht konstant, und insbesondere die Entwicklung des Risikokapitalmarktes, der Hauptfinanzierungsquelle für diese Firmen, ist in hohem Maße zyklisch (Gompers, Lerner, 1999). Demzufolge werden die Unternehmen eine höhere Neigung zu strategischen Allianzen als Alternative zur Ressourcenakquisition über den Kapitalmarkt zeigen, wenn dieser sich in einem Abwärtstrend befindet.

\section{Einflussstärke der Faktoren}

Obige Argumentation zeigt, dass ein Defizit ihrer Firma an (i) finanziellen Ressourcen, (ii) Produktkandidaten in frühen Entwicklungsstadien, (iii) Produktkandidaten in späten Entwicklungsstadien, (iv) Patenten, (v) qualifizierten Wissenschaftlern und (vi) Netzwerkkontakten für Biotechnologiemanager einen Anreiz darstellt, neue Allianzpartner zu suchen. Zudem sind (vii) hoher Konkurrenzdruck und (viii) fehlende Möglichkeiten zur Kapitalakquisition Triebkräfte der Allianzsuche. Es ist jedoch zu vermuten, dass der Einfluss all dieser Faktoren nicht gleich stark ist, da Entscheidungsfaktoren gewöhnlich 
von Managern unterschiedlich gewichtet werden. Beispielsweise berücksichtigen Risikokapitalmanager bei Investitionsentscheidungen die unterschiedlichen Eigenschaften potentieller Investmentprojekte unterschiedlich stark (Shepherd, 1999). Pablo (1994) zeigte, dass unterschiedliche Eigenschaften akquirierter Firmen die strategische Entscheidung von Managern über den Integrationsgrad unterschiedlich stark beeinflussen. Schließlich fanden Hitt et al. (2004), dass die Gewichtung von Entscheidungsfaktoren bei der Selektion von Allianzpartnern differiert. Diese Befunde legen folgende explorative Forschungsfrage hinsichtlich der Einflussstärke der oben abgeleiteten Faktoren nahe:

Welche relative Einflussstärke haben ein Defizit an (i) finanziellen Ressourcen, (ii) Produktkandidaten in frühen Entwicklungsstadien, (iii) Produktkandidaten in späten Entwicklungsstadien, (iv) Patentschutz, (v) qualifizierten Wissenschaftlern und (vi) Netzwerkkontakten sowie (vii) Konkurrenzdruck und (viii) fehlende Möglichkeiten zur Kapitalakquisition auf den Anreiz für Manager eines Biotechnologieunternehmens, neue Allianzpartner suchen?

\section{Forschungsmethodik}

\section{Datensammlung und Beschreibung der Stichprobe}

Der Untersuchungsrahmen dieser Studie sind Manager junger Firmen in der deutschen Biotechnologiebranche. Das Beratungsunternehmen Ernst \& Young, das einen auf Umfragen basierenden jährlichen Report über die Branche herausgibt, führt auf einem dem Report des Jahres 2003 beigelegten Poster 212 Firmen auf, die an der Umfrage im Jahr 2002 teilgenommen haben. Da diese Biotechnologieunternehmen jedoch in einer Vielzahl unterschiedlicher Geschäftsfelder tätig sind, was sich auf ihre Finanzierungsquellen, Marktstrategien, und F\&E-Prozesse auswirkt, wurden für diese Studie in Anlehnung an existierende Arbeiten (Deeds et al., 1998; Deeds, Hill, 1996) nur Firmen berücksichtigt, die Medikamente oder Diagnostika entwickeln. Damit bestand der Untersuchungsrahmen aus 99 biopharmazeutischen Unternehmen, von denen zwölf zum Zeitpunkt der Untersuchung im Jahre 2005 bereits ihre Geschäftstätigkeit eingestellt hatten.

Alle verbleibenden 87 Firmen wurden im Frühjahr 2005 per Telefon kontaktiert und um die Teilnahme eines Mitglieds des Top-Managements in der Studie gebeten. Als Top-Management wurden dabei alle Vorstände und Vize-Präsidenten definiert (Deeds et al., 1999). Zudem wurden leitende Business-Development-Manager einbezogen, da diese in einer Vielzahl der Firmen für die Knüpfung und Abwicklung strategischer Allianzen verantwortlich sind. Manager aus 68 Firmen erklärten sich zu einer Teilnahme bereit.

Außer den ersten sechs Firmen, die die Studie in Gegenwart eines Autors dieser Arbeit durchführten, wurden allen Firmen die Experimentbögen in schriftlicher Form zugeschickt. Falls nach vier Wochen noch kein Rücklauf der Bögen zu verzeichnen war, wurden die Manager erneut angerufen und um Teilnahme gebeten. 52 Manager aus 45 Firmen schickten schließlich ausgefüllte Experimente zurück, was einer relativ hohen 
Rücklaufquote von 52 \% (bezogen auf die kontaktierten Firmen) entspricht. Da sich die Ergebnisse der Studie nicht wesentlich verändern, wenn nur ein Manager pro Firma berücksichtigt wird, werden im Folgenden nur die Ergebnisse für die gesamte Stichprobe berichtet.

Im Durchschnitt waren die teilnehmenden Manager 41,6 Jahre alt, hatten 8,0 Jahre Erfahrung in der Biotechnologieindustrie und 5,7 Jahre Erfahrung in Top-ManagementPositionen. 67 \% der Teilnehmer waren in CEO/CFO/CSO-Positionen, 82 \% hatten eine naturwissenschaftliche (Biologie, Biochemie, Chemie, Medizin) und $18 \%$ eine wirtschaftswissenschaftliche Ausbildung. Die Firmen waren durchschnittlich 6,7 Jahre alt und beschäftigten 50 Mitarbeiter.

\section{Conjoint-Experiment und Variable}

In dieser Studie wurde ein Conjoint-Experiment verwendet um den Anreiz für Biotechnologiemanager, strategische Allianzen zu suchen, zu analysieren. Conjoint-Experimente basieren auf der Annahme, dass Entscheidungen von Individuen in grundlegende Strukturen zerlegt werden können (Green, 2001). Conjoint-Studien werden in Forschungsarbeiten in den Gebieten Marketing, Psychologie, Strategisches Management, sowie vieler anderer Disziplinen verwendet (Wittink, Cattin, 1989). Auch für die Forschung an strategischen Allianzen wurden sie eingesetzt, insbesondere um den Einfluss von Partnereigenschaften auf die Allianzentscheidung zu untersuchen (Dollinger et al., 1997).

In einem Conjoint-Experiment beurteilt ein Individuum verschiedene Szenarien, von denen jedes anhand einer Anzahl von Parametern beschrieben wird. Diese Parameter haben unterschiedliche, für jedes Szenario festgelegte Ausprägungen (z. B. hoch und niedrig). Die Parameter stellen die unabhängigen Forschungsvariablen dar und die Beurteilung die abhängige Variable.

Als abhängige Variable wurde der Anreiz der Manager, neue Allianzpartner zu suchen, verwendet. Dabei wurden strategische Allianzen als jede Art von Firmenkooperationen, die der Produktentwicklung dienen, definiert (Deeds, Hill, 1996). Die Manager wurden gebeten, Beurteilungen auf einer 7-Punkte-Likert-Skala, deren Endpunkte mit "sehr niedriger Anreiz, eine strategische Allianz zu suchen" und "sehr hoher Anreiz, eine strategische Allianz zu suchen” bezeichnet waren, abzugeben.

Die unabhängigen Variablen wurden in jedem Szenario durch acht Ressourcen-/Umweltparameter dargestellt, die je zwei mögliche Ausprägungen besaßen. Liquidität des Unternehmens bezeichnete seine Ausstattung an kurzfristig verfügbaren finanziellen Ressourcen und hatte die Ausprägungen hoch (beachtliche Liquiditätsreserven für Fortbestand und Wachstum in den nächsten Jahren) und niedrig (beschränkte Liquiditätsreserven, die voraussichtlich binnen Jahresfrist erschöpft sind). Frühe Produkte bezeichnete die Zahl der Produktkandidaten, die sich in frühen Entwicklungsstadien befinden, und hatte die Ausprägungen hoch (Entwicklungspipeline weist eine beachtliche Anzahl an Produkten in frühen Entwicklungsphasen auf) und niedrig (Entwicklungspipeline weist nur wenige Produkte in frühen Entwicklungsphasen auf.). Späte Produkte bezeichnete die Zahl der Produktkandidaten, die sich in fortgeschrittenen Entwicklungsstadien befinden, und hatte die Ausprägungen hoch (Entwicklungspipeline weist eine beachtliche $\underline{\text { Anzahl an Produkten in späten Entwicklungsphasen auf) und niedrig (Entwicklungspipe- }}$ 
line weist nur wenige Produkte in späten Entwicklungsphasen auf). Patente bezeichnete die Anzahl der Patente und hatte die Ausprägungen hoch (breites Portfolio an gesicherten Patenten) und niedrig (beschränktes Portfolio an Patenten, die zudem durch Patentstreitigkeiten unsicher sind). Team bezeichnete die Qualität des wissenschaftlichen Teams und hatte die Ausprägungen hoch (Team besteht aus herausragenden und renommierten Spezialisten) und niedrig (Team besteht aus nur durchschnittlichen Wissenschaftlern). Netzwerk bezeichnete die Größe des Kontaktnetzwerks und hatte die Ausprägungen ausgedehnt (ausgedehntes Kontaktnetzwerk mit anderen Firmen, Forschungseinrichtungen und Investoren) und beschränkt (nur wenige Kontakte zu anderen Firmen, Forschungseinrichtungen und Investoren). Finanzierungsumfeld bezeichnete die Möglichkeiten, Kapital zu akquirieren und hatte die Ausprägungen attraktiv (gute Möglichkeiten, Risikokapital zu akquirieren oder an die Börse zu gehen) und unattraktiv (nur beschränkte Möglichkeiten, Risikokapital zu akquirieren; das Börsenfenster ist geschlossen). Wettbewerb bezeichnete die Konkurrenzsituation der Firma und hatte die Ausprägungen hoch (sehr kompetitive Projekte und unmittelbare Konkurrenz zu mehreren Firmen) und niedrig (wenig kompetitive Projekte und keine unmittelbare Konkurrenz zu anderen Firmen).

\section{Experimentelles Design, Reliabilität and Externe Validität}

Die Szenarien des Experiments bestanden aus acht Parametern, von denen jeder zwei mögliche Ausprägungen besaß, woraus sich $2^{8}=256$ mögliche Kombinationen ergaben. Um diese Zahl auf 16 zu reduzieren wurde ein orthogonales faktorielles Design verwendet (Hahn, Shapiro, 1966). Zur Überprüfung der Reliabilität der Antworten wurden Test-Retest-Checks durchgeführt (Shepherd, Zacharakis, 1997) womit sich insgesamt 32 Szenarien ergaben. Um Anordnungseffekte der Parameter in den Szenarien oder der Szenarienabfolge auszuschließen wurden sowohl Parameter als auch Szenarien in jeweils zwei unterschiedliche, zufällige Reihenfolgen gebracht. Damit ergaben sich vier Versionen des Experiments, die zufällig zwischen den Teilnehmern verteilt wurden. Es konnten keine signifikanten Unterschiede im Antwortverhalten festgestellt werden. Den 32 Szenarien wurde noch ein "Übungsszenario" vorangestellt, das die Manager mit dem Experiment vertraut machen sollte und nicht in die statistische Auswertung einbezogen wurde.

Zwei möglichen Kritikpunkten sind Conjoint-Experimente ausgesetzt. Zum einen wird manchmal argumentiert, dass die Teilnehmer nur deshalb ihre Einschätzungen anhand der vorgestellten Parameter treffen, weil sie Teil des Experiments sind (Shepherd, Zacharakis, 1997). Dieser Kritik wurde auf mehrfache Weise begegnet. Zum einen wurden im Vorfeld des Experiments vier Interviews mit Biotechnologiemanagern geführt. Zweitens wurden die ersten sechs Experimente im Beisein eines Mitglieds des Forscherteams durchgeführt um Feedback zum Experiment und den verwendeten Variablen zu erhalten. Von allen Interviewpartnern und Teilnehmern wurde die Relevanz der verwendeten Entscheidungsparameter bestätigt. Schließlich kann von hoher externer Validität der Ergebnisse ausgegangen werden, da alle Parameter aus der Literatur abgeleitet wurden (Shepherd, Zacharakis, 1997).

Ein weiterer möglicher Kritikpunkt von Conjoint-Experimenten ist, dass diese „Papier- und Bleistiftexperimente“ (i) Teilnehmer dazu zwingen, Entscheidungen anhand 
einiger weniger Parameter abzugeben, was Situationen des wirklichen Lebens nur unzureichend beschreibt, (ii) nicht genügend unmittelbare und emotionale Einbindung in die Entscheidungssituation gewährleisten, und (iii) Teilnehmer möglicherweise Kriterien nur deshalb als entscheidungsrelevant betrachten, weil sie im Experiment dargestellt werden. Der Reduktionismus von Conjoint-Experimenten ist jedoch konsistent mit der Beobachtung, dass sowohl Laien als auch Experten gewöhnlich nur drei bis sieben Kriterien in ihre Entscheidungsfindung im realen Leben einbeziehen (Stewart, 1988). Des weiteren haben Studien gezeigt, dass auch in den abstaktesten Situationen Conjoint-Analysen das reale Entscheidungsverhalten von Individuen gut abbilden (Brown, 1972; Hammond, Adelman, 1976). Schließlich ist es unwahrscheinlich, dass die Teilnehmer an der vorliegenden Studie den Kriterien nur aufgrund ihrer Einbeziehung in das Experiment Beachtung schenken, da alle Teilnehmer signifikante Erfahrung mit realen, ähnlichen Entscheidungssituationen aufweisen (Brehmer, Brehmer, 1988), wie aus den obigen Werten für die Branchen- und Managementerfahrung der Teilnehmer entnommen werden kann.

\section{Analyse und Ergebnisse}

Die Reliabilität der Antworten wurde mittels Pearson-Korrelationen der Test und RetestSzenarien untersucht. Für 78 \% der Teilnehmer waren die Korrelationen statistisch signifikant $(\mathrm{p}<0,05)$, was leicht unter den Angaben vergleichbarer Studien liegt (Shepherd, 1999: 92 \%). Die mittlere Korrelation betrug jedoch 0,67, was mit Literaturwerten übereinstimmt (Shepherd, 1999: 0,69) und bestätigt, dass die Teilnehmer das Experiment konsistent bearbeitet haben.

Die statistische Analyse der Daten erfolgte analog der Studie von Shepherd (1999), der die Einflussstärke der Faktoren der Investitionsentscheidungen von Risikokapitalmanagern untersuchte. Zunächst wurden für jeden Teilnehmer Regressionskoeffizienten der Parameter ermittelt, um die Entscheidungen der Individuen in ihre grundlegenden Strukturen zu zerlegen. 84 \% der individuellen Entscheidungsmodelle waren statistisch signifikant $(\mathrm{p}<0,05)$ (Shepherd, 1999: 75 \%). Das mittlere $\mathrm{R}^{2}$ dieser Modelle betrug 0,79 (Shepherd, 1999: 0,78). Die über alle Teilnehmer gemittelten Regressionskoeffizienten stellen ein Maß für das Entscheidungsverhalten der Stichprobe dar, wobei die Vorzeichen der Koeffizienten die Richtung des Einflusses angeben. Im nächsten Schritt wurde analog Healy et al. (1987) mittels einer Z-Statistik überprüft, ob die über die Stichprobe gemittelten Koeffizienten das Entscheidungsverhalten statistisch signifikant beschreiben. Zudem wurden die Standardabweichungen und die obere und untere Quartile der Koeffizienten berechnet, um ein Maß für die Heterogenität der Entscheidungen zu erhalten. Die Schiefe gibt an, inwiefern die Koeffizientenverteilung über die Teilnehmer von einer Normalverteilung abweicht. Die Ergebnisse sind in Tabelle 1 dargestellt.

Die Z-Werte in Tabelle 1 zeigen, dass alle Entscheidungsparameter mit Ausnahme der Netzwerkgröße signifikant das Entscheidungsverhalten von Biotechnologiemanagern, Allianzen zu suchen, beschreiben. Die Vorzeichen der Koeffizienten entsprechen den aus obiger Argumentation abgeleiteten Richtungen: Manager von Unternehmen mit geringer Liquidität, wenigen frühen und späten Produktkandidaten, schwachem Patentschutz 
Tab. 1. Entscheidungskriterien für die Allianzsuche

\begin{tabular}{|l|c|c|c|c|c|c|}
\hline Parameter & $\begin{array}{c}\text { Koeffizient } \\
\text { (Mittelwert) }\end{array}$ & Z-Wert & $\begin{array}{c}\text { Standard- } \\
\text { abweichung }\end{array}$ & $\begin{array}{c}\text { Obere } \\
\text { Quartile }\end{array}$ & $\begin{array}{c}\text { Untere } \\
\text { Quartile }\end{array}$ & Schiefe \\
\hline Ressourcen \\
\hline Liquidität & $-1,354$ & $-25,780^{* * *}$ & 1,342 & $-0,531$ & $-2,407$ & 0,266 \\
\hline Frühe Produkte & $-0,395$ & $-7,594 * * *$ & 0,656 & 0,000 & $-0,860$ & 0,447 \\
\hline Späte Produkte & $-0,558$ & $-11,437 * * *$ & 1,029 & 0,282 & $-1,235$ & 0,189 \\
\hline Patente & $-0,277$ & $-5,591^{* * *}$ & 0,493 & 0,054 & $-0,605$ & $-0,454$ \\
\hline Team & $-0,132$ & $-2,769 * *$ & 0,398 & 0,172 & $-0,395$ & 0,309 \\
\hline Netzwerk & 0,040 & 0,601 & 0,389 & 0,250 & $-0,250$ & 0,295 \\
\hline Umwelt & \multicolumn{7}{|l|}{} & & & \\
\hline Finanzierung & $-0,989$ & $-19,433^{* * *}$ & 0,817 & $-0,386$ & $-1,625$ & $-0,324$ \\
\hline Wettbewerb & 0,204 & $3,584 * * *$ & 0,466 & 0,492 & $-0,125$ & 0,655 \\
\hline
\end{tabular}

${ }^{*} \mathrm{p}<0,05 ; * * \mathrm{p}<0.01 ; * * * \mathrm{p}<0,001 ; \mathrm{n}=1664$ Entscheidungen von 52 Managern

sowie einer niedrigen Teamqualität suchen verstärkt nach Allianzpartnern. Der Anreiz zur Suche wird zudem durch ein wenig attraktives Finanzierungsumfeld und erhöhten Wettbewerbsdruck verstärkt.

Analog zu der Studie von Shepherd (1999) wurde als Maß für die Einflussstärke der einzelnen Parameter das von Hays (1973) beschriebene $\omega^{2}$ verwendet. $\omega^{2}$ ist ein Maß für die Varianz, die im Antwortverhalten der Teilnehmer durch die Parameter erklärt wird. Grundlage der $\omega^{2}$-Berechnung bildet eine ANOVA-Analyse der individuellen Teilnehmer. Aus den Ergebnissen wird dann für jeden Teilnehmer und jeden Parameter ein $\omega^{2}$ berechnet. Die erhaltenen $\omega^{2}$-Werte werden wiederum über alle Teilnehmer gemittelt. Diese Mittelwerte sind in Tabelle 2 angegeben.

Die Standardabweichungen und die oberen und unteren Quartilen der Regressionskoeffizienten (Tabelle 1) zeigen, dass durchaus Heterogenität im Entscheidungsverhalten zwischen den Managern zu beobachten ist. Deshalb wurden die $\omega^{2}$ paarweise mittels Mann-Whitney-Tests verglichen um zu überprüfen, ob die Unterschiede statistisch signifikant sind (Tabelle 2). Diese Form nicht-parametrischer Tests hat gegenüber t-Tests den Vorteil, dass eine Normalverteilung der $\omega^{2}$ keine Voraussetzung ist. Da die Werte der Schiefe der Koeffizienten (Tabelle 1) zeigen, dass eine Normalverteilung der Antworten (Schiefe $=0$ ) nur in grober Näherung vorliegt und eine visuelle Inspektion der $\omega^{2}$ diesen Eindruck bestätigt sind Mann-Whitney-Tests im vorliegenden Fall angemessen für paarweise Vergleiche der Einflussstärken.

Die Werte in Tabelle 2 zeigen, dass die Liquidität der Firma die größte Einflussstärke aufweist $(0,239)$ und diese Einflussstärke auf statistisch signifikantem Niveau größer ist als alle anderen Einflussstärken. Zudem ist zu erkennen, dass das Fehlen von Produkten in späten Entwicklungsstadien $(0,119)$ sowie das Finanzierungsumfeld $(0,116)$ ähnliche, aber signifikant größere Einflussstärken aufweisen als die anderen Entscheidungsparameter (mit Ausnahme der Liquidität). Die Einflussstärken für frühe Produkte $(0,041)$, Patente $(0,018)$, das wissenschaftliche Team $(0,003)$, das Netzwerk $(0,002)$ sowie den Wettbewerb $(0,005)$ sind deutlich und statistisch signifikant niedriger. Zusammenfassend 
Tab. 2. Relative Bedeutung der Entscheidungskriterien

\begin{tabular}{|c|c|c|c|c|c|c|c|c|c|}
\hline \multirow[b]{2}{*}{ Parameter } & \multirow{2}{*}{$\begin{array}{c}\omega^{2} \\
\text { Mittel- } \\
\text { wert }\end{array}$} & \multicolumn{8}{|c|}{ Paarweise Vergleiche (Mann-Whitney-Tests) } \\
\hline & & Liqu. & $\begin{array}{l}\text { Frühe } \\
\text { Prod. }\end{array}$ & $\begin{array}{l}\text { Späte } \\
\text { Prod. }\end{array}$ & Patente & Team & $\begin{array}{l}\text { Netz- } \\
\text { werk }\end{array}$ & Finanz & $\begin{array}{c}\text { Wettbe- } \\
\text { werb }\end{array}$ \\
\hline Liquidität & 0,239 & & $442 * * *$ & $854 * *$ & $307 * * *$ & $197 * * *$ & $189 * * *$ & $842 * *$ & $212 * * *$ \\
\hline Frühe Prod. & 0,041 & $442 * * *$ & & $725 * * *$ & 1110 & $989 *$ & $946 * *$ & $835 * *$ & $947 *$ \\
\hline Späte Prod. & 0,119 & $854 * *$ & $725 * * *$ & & $526 * * *$ & $352 * * *$ & $315^{* * * *}$ & 1292 & $357 * * *$ \\
\hline Patente & 0,018 & $307 * * *$ & 1110 & $526 * * *$ & & 1264 & 1198 & $632 * * *$ & 1208 \\
\hline Team & 0,003 & $197 * * *$ & $989 *$ & $352 * * *$ & 1264 & & 1288 & $497 * * *$ & 1299 \\
\hline Netzwerk & 0,002 & $189 * * *$ & $946^{* *}$ & $315^{* * *}$ & 1198 & 1288 & & $470 * * *$ & 1326 \\
\hline Finanzierung & 0,116 & $842 * *$ & $835^{* *}$ & 1292 & $632 * * *$ & $497 * * *$ & $470 * * *$ & & $503 * * *$ \\
\hline Wettbewerb & 0,005 & $212 * * *$ & $947^{*}$ & $357 * * *$ & 1208 & 1299 & 1326 & $503 * * *$ & \\
\hline
\end{tabular}

${ }^{*} \mathrm{p}<0,05 ;{ }^{* *} \mathrm{p}<0.01 ; * * * \mathrm{p}<0,001 ; \mathrm{n}=1664$ Entscheidungen von 52 Managern

bedeutet dies, dass die Allianzsuche von Biotechnologiemanagern in erster Linie von fehlender Liquidität und in zweiter Linie von fehlenden Finanzierungsmöglichkeiten und fehlenden späten Produktentwicklungskandidaten getrieben wird, wohingegen andere Ressourcen- und Umweltparameter nur untergeordnete Rollen spielen.

Eine interessante Beobachtung ergibt sich aus der Heterogenität der Regressionsparameter in Tabelle 1. So weisen die oberen und unteren Quartilen für die Parameter späte Produkte, Patente, Team, Netzwerk und Wettbewerb Vorzeichenwechsel auf. Auch für die anderen Parameter wie z. B. Liquidität und frühe Produkte sind die Standardabweichungen im Vergleich mit den absoluten Werten der Koeffizienten relativ groß. Dieser Effekt könnte auf Inkonsistenzen im Antwortverhalten zurückzuführen sein; es ist jedoch auch möglich, dass im Antwortverhalten der Manager zwei Gruppen unterscheidbar sind - manche Manager werden eher durch ein Fehlen, andere eher durch ein Vorhandensein von Ressourcen zur Allianzsuche motiviert. Diese Annahme wurde mittels einer k-MeansClusteranalyse näher untersucht. Das Verfahren erlaubt es, die Untersuchungsobjekte in Gruppen optimaler Homogenität einzuteilen.

Voraussetzung der Durchführung einer k-Means-Clusteranalyse ist, dass die Anzahl der zu bildenden Cluster bekannt ist. Obige Argumentation legt eine Clusterzahl von zwei (Allianz-Triebkraft vorhandene vs. fehlende Ressourcen) nahe. ${ }^{1}$ Darüber hinaus sollten für die k-Means-Analyse initiale Anhaltspunkte für die Lage der Clusterzentren gegeben sein. Da die Clusterzahl aus den Vorzeichenwechseln der oberen und unteren Quartilen der Regressionskoeffizienten abgeleitet wurde, bietet es sich an, diese Quartilen (Tabelle 1) als Startpunkte für die Clusteranalyse zu verwenden. ${ }^{2}$ Zudem wurde eine ANOVA-Analyse durchgeführt, die als Anhaltspunkt für die Distanz zwischen den Clusterzentren dienen kann. Tabelle 3 zeigt die Ergebnisse der Analyse.

Aus Tabelle 3 ist ersichtlich, dass sich für Liquidität und späte Produkte zwei Cluster mit Schwerpunkten unterschiedlicher Vorzeichen ergeben. Zudem zeigen die hohen FWerte und niedrigen p-Werte der ANOVA, dass die Clusterzentren für beide Parameter weit voneinander entfernt liegen. Dies deutet darauf hin, dass für manche Manager (Cluster 1) vorhandene Liquidität und späte Produkte als Treiber für die Allianzsuche 
Tab. 3. k-Means-Clusteranalyse

\begin{tabular}{|l|c|c|c|c|}
\hline & \multicolumn{2}{|c|}{ Clusteranalyse } & \multicolumn{2}{c|}{ ANOVA } \\
\hline Parameter & $\begin{array}{c}\text { Cluster 1 } \\
\text { Mittelwert }\end{array}$ & $\begin{array}{c}\text { Cluster 2 } \\
\text { Mittelwert }\end{array}$ & F-Wert & p-Wert \\
\hline Liquidität & 0,14 & $-2,02$ & 63,865 & 0,000 \\
\hline Frühe Produkte & $-0,37$ & $-0,41$ & 0,047 & 0,830 \\
\hline Späte Produkte & 0,10 & $-0,85$ & 11,208 & 0,002 \\
\hline Patente & $-0,16$ & $-0,33$ & 1,359 & 0,249 \\
\hline Team & $-0,03$ & $-0,18$ & 1,692 & 0,199 \\
\hline Netzwerk & 0,00 & 0,06 & 0,224 & 0,638 \\
\hline Finanzierung & $-0,43$ & $-1,24$ & 13,343 & 0,001 \\
\hline \multicolumn{2}{|c|}{ Wettbewerb } & 0,03 & 3,568 & 0,065 \\
\hline \multicolumn{2}{l|}{ Objekte in Cluster 1: 16; Objekte in Cluster 2: 36; Initiale Clusterzentren: obere und untere Quartilen aus } \\
Tabelle 1; Quadrierte Euklidische Distanz; Software SPSS; Konvergenz nach drei Iterationen
\end{tabular}

fungieren, wohingegen für die Mehrheit (Cluster 2) fehlende Liquidität und fehlende späte Produkte eher eine Motivation zur Allianzsuche darstellen. Für die anderen Entscheidungsparameter konnten keine Vorzeichenunterschiede der Clusterzentren festgestellt werden, was andeutet, dass die Vorzeichenwechsel der oberen und unteren Quartilen eher auf Inkonsistenzen als auf systematische Unterschiede im Antwortverhalten zurückzuführen sind. Diese Befunde werden im Folgenden diskutiert.

\section{E. Diskussion und Implikationen}

Ziel dieser Arbeit war es, die Einflussfaktoren der Allianzsuche von Biotechnologiemanagern zu untersuchen. Aus einer ressourcenorientierten Perspektive wurden verschiedene Einflussfaktoren identifiziert, die sowohl die Ressourcenausstattung der Unternehmen als auch die Ressourcenanforderungen und -akquisitionsmöglichkeiten der Umwelt beschreiben. Die Relevanz und Einflussstärke der Entscheidungsfaktoren wurden mittels eines Conjoint-Experiments überprüft.

Die Ergebnisse dieser Studie zeigen, dass eine geringe Ausstattung an finanziellen, Produkt-, Patent- und Humanressourcen entscheidend für die Allianzsuche von Managern sind. Manager versuchen, fehlende Ressourcen durch Allianzbildung zu akquirieren. Zudem berücksichtigen die Manager, welche Ressourcenanforderungen die Umwelt an ihre Firma stellt bzw. welche Möglichkeiten zur Ressourcenakquisition sie bietet. Damit können existierende Studien in der Literatur zu strategischen Allianzen bestätigt und erweitert werden, die allgemein den Wunsch, Ressourcen von Allianzpartner zu akquirieren, beschreiben (Das, Teng, 2000) und zeigen, dass dies insbesondere in „strategisch verwundbaren Ressourcenpositionen“ (Eisenhardt, Schoonhoven, 1996), also bei starker Konkurrenz, der Fall ist.

Ein wichtiger Beitrag dieser Studie ist, dass sie einen Einblick in die relative Bedeutung von Einflussfaktoren der Allianzsuche erlaubt. Bisherige Arbeiten haben sich darauf 
beschränkt, Motive wie die Teilung von Entwicklungsrisiken (Hamel et al., 1989), die Gewinnung von Marktmacht (Hagedoorn, 1993), oder eine Erhöhung der Legitimität und Visibilität des Unternehmens (Baum, Oliver, 1991) zu identifizieren oder zu untersuchen, wie Eigenschaften von Ressourcen im Allgemeinen die Neigung von Unternehmen zur Allianzbildung beeinflussen (Das, Teng, 2000). In dieser Arbeit wurde explizit in einem spezifischen Kontext - Produktentwicklungsallianzen junger Biotechnologieunternehmen - eine relative Bedeutung von Motiven ermittelt, indem die Einflussstärke von Entscheidungsfaktoren auf die Allianzsuche von Managern berechnet wurde.

Ein Ergebnis dieser Arbeit ist, dass die Einflussstärke geringer Liquidität die aller anderen Faktoren dominiert. Je weniger finanzielle Ressourcen ein Biotechnologieunternehmen besitzt, desto stärker die Neigung der Manager zur Allianzsuche. Da finanzielle Ressourcen fungibel sind und daher verwendet werden können, um andere Ressourcen über die Faktormärkte zu akquirieren (Dollinger, 1995), zeigt das Ergebnis die Präferenz von Managern für die interne Weiterentwicklung der Ressourcenbasis des Unternehmens im Falle vorhandener Liquidität im Gegensatz zu einer externen Entwicklung in Form von Allianzen. Diese Präferenz ist im Einklang mit dem theoretischen Modell der optimalen Kapitalstruktur von Myers und Majluf (1984), nach dem die Informationsasymmetrien zwischen den Managern einer potentiell allianzsuchenden Firma und einer Partnerfirma eine Allianz oftmals zu teuer machen. Zudem bedeutet eine Allianz immer die Gefahr opportunistischen Partnerverhaltens und damit verbundenem Verlust von firmeninternem Wissen und anderen Ressourcen (Deeds, Hill, 1998). Im Falle einer niedrigen Liquidität ihres Unternehmens hingegen sind die Manager jedoch gezwungen, nach neuen Allianzpartnern zu suchen, da anderenfalls das Überleben ihres Unternehmens nicht gesichert werden kann. In diesen Situationen sehen sie Allianzen offenbar als effiziente Mittel zur Kostensenkung und Risikominimierung (Eisenhardt, Schoonhoven, 1996) und sind bereit, die Risiken opportunistischen Partnerverhaltens einzugehen und hohe Preise für eine Allianz zu bezahlen (Lerner et al., 2003).

Des Weiteren zeigen die Einflussstärken der Entscheidungsfaktoren, dass die Allianzsuche in starkem Maße von fehlenden Produktkandidaten in späten Entwicklungsstadien sowie mangelnden Möglichkeiten zur Kapitalakquisition getrieben wird. Beide Entscheidungsfaktoren überstiegen in ihren Einflussstärken auf statistisch signifikantem Niveau alle anderen mit Ausnahme der Liquidität. Für die Bedeutung fehlender Möglichkeiten zur Kapitalakquisition lässt sich eine analoge Begründung zur Liquidität anführen: Manager bevorzugen eigene finanzielle Ressourcen, die sie über den Kapitalmarkt akquirieren können, gegenüber Allianzen für die Weiterentwicklung der Ressourcenbasis ihres Unternehmens. Fehlt der Zugang zu externem Kapital, werden höhere Preise und Gefahren eines opportunistischen Verhaltens des Partners in Kauf genommen (Lerner et al., 2003). Die hohe Bedeutung fehlender Produktkandidaten in späten Entwicklungsstadien für die Allianzsuche ist dadurch zu erklären, dass die Bewertung eines Biotechnologieunternehmens stark an das Vorhandensein einer „reifen“ Produktpipeline gekoppelt ist (Rothaermel, Deeds, 2004). Darüber hinaus benötigen späte Produktkandidaten weniger Ressourcen zu ihrer Entwicklung zur Marktreife als frühe Kandidaten und bergen ein geringeres Risiko des Scheiterns (DiMasi et al., 2003). Vor allem aber bieten späte Produktkandidaten eine Aussicht auf baldige Generierung eines Cash-Flow, der dem Unternehmen Liquidität sichern kann und damit die Überlebenswahrscheinlichkeit erhöht. 
Selbst wenn ein Biotechnologieunternehmen durch die Markteinführung des ersten Produktes oftmals noch nicht profitabel wird, erhöht sich die Wahrscheinlichkeit weiterer Kapitalakquisition von Investoren hierdurch erheblich (Deeds et al., 2004).

Eine genauere Analyse der Heterogenität des Antwortverhaltens der Manager ergab jedoch, dass trotz der hohen Einflussstärke eines Defizits an Liquidität und späten Produktkandidaten für die gesamte Stichprobe ein nicht unerheblicher Anteil der Manager eher vorhandene als fehlende Ressourcen als eine Triebkraft zur Allianzsuche ansieht. Im Falle später Produktkandidaten wäre eine mögliche Erklärung, dass manche Biotechnologieunternehmen nicht über die nötigen Kompetenzen für die Fertigstellung der Entwicklung (z. B. späte klinische Prüfungsphasen oder Zulassungsverfahren) verfügen und deshalb nach Allianzpartnern Ausschau halten (Audretsch, Feldman, 2003). Für den Fall vorhandener Liquidität könnte sich eine mögliche Erklärung aus der stärkeren Verhandlungsposition der Manager bei der Allianzbildung ergeben. Arbeiten von Lerner und Merges (1998) haben gezeigt, dass etablierte Pharmaunternehmen eine schwache Liquiditätsposition von Biotechnologieunternehmen dazu ausnutzen, ihnen strenge Kontrollen im Allianzvertrag aufzuzwingen. Diese strengen Kontrollen führen allerdings dazu, dass die Erfolgsaussichten der Allianz deutlich sinken. Somit könnten manche Biotechnologiemanager eine neue Allianz nur dann als erfolgsversprechend einstufen, wenn sie genügend Liquidität besitzen. Ebenso ist es möglich, dass die Manager generell die Abgabe von zu vielen Kontrollrechten vermeinden wollen und deshalb nur bei hinreichender Liquidität auf Partnersuche gehen. Insgesamt stützen und erweitern diese Befunde existierende theoretische Arbeiten, die postulieren, dass sowohl fehlende als auch vorhandene Ressourcen eine Motivation zur Allianzsuche darstellen können (Das, Teng, 2000).

Aus den Ergebnissen der Studie ergeben sich Implikationen für die Managerpraxis. Aus Perspektive der allianzsuchenden Firma kann es für die Manager hilfreich sein, ihre Entscheidungen zur Allianzsuche besser zu verstehen und aufgrund dessen bessere und genauere Entscheidungen zu treffen. Da Allianzen meist langfristige Bindungen zwischen Unternehmen darstellen und Ressourcen der Unternehmen für lange Zeit binden (Das, Teng, 2000), sollten Manager sich genau überlegen, Allianzpartner zu suchen. Insbesondere wenn mangelnde Liquidität die Haupttriebkraft hinter der Allianzentscheidung ist, kann die Allianz und deren Ressourcenanforderung eine Bedrohung für die Firma darstellen (Alvarez, Barney, 2001). Aus der Perspektive der Firma, die um eine Allianz ersucht wird, ist es ebenso wichtig die Motive des suchenden Partners zu verstehen. Im Einklang mit existierenden Arbeiten von Lerner und Kollegen (Lerner et al., 2003) zeigen die Resultate dieser Arbeit, dass, wenn die suchende Firma wenige finanzielle Ressourcen besitzt und das Finanzierungsumfeld unattraktiv ist, die Zielfirma in einer starken Verhandlungsposition ist. Allerdings besteht die Möglichkeit, dass der suchende Partner nach Erhalt einer Zahlung im Zuge der Allianz oder bei sich ändernden Kapitalmärkten sich opportunistisch verhalten und seinen Teil zur Erfüllung der Allianz nicht beitragen wird. 


\section{F. Limitationen and zukünftige Forschung}

Die Limitationen dieser Studie bieten Möglichkeiten für weiterführende Forschungsarbeiten. Zunächst ist festzuhalten, dass im Rahmen dieser Arbeit nur direkte Einflüsse der Ressourcensituation und Umwelt auf die Allianzsuche von Managern untersucht werden, potentielle Interaktionen der Entscheidungsparameter jedoch keine Berücksichtigung finden. Es ist allerdings möglich, dass z. B. zwischen den finanziellen Ressourcen eines Unternehmens und dem Einfluss des Finanzierungsumfeldes Interaktionseffekte auftreten - hinreichende finanzielle Ressourcen ermöglichen es Managern, Zeiten knapper Kapitalmärkte auch ohne Allianzen zu überstehen und abzuwarten bis sich die Kapitalmärkte erneut öffnen. Zudem ist es z. B. möglich, dass vorhandene finanzielle Ressourcen eine Allianzsuche mit dem Ziel, fehlende Produktkandidaten zu akquirieren, attraktiver machen, da in diesem Fall genügend Liquidität zur Weiterentwicklung der Kandidaten vorhanden ist. Eine Untersuchung dieser Interaktionseffekte stellt eine wichtige Herausforderung weiterführender Arbeiten dar.

Zweitens ist anzumerken, dass die Anwendung der metrischen Conjoint-Analyse und der dabei zugrunde liegende linear-kompensatorische Ansatz nicht berücksichtigen, dass die Sicherung der Liquidität für Manager eine notwendige Bedingung für eine Allianzsuche darstellen könnte. So ist es vorstellbar, dass Manager nur dann auf Allianzsuche gehen, wenn ihr Unternehmen über genügend Liquidität verfügt um die Allianz auch zu einem erfolgreichen Abschluss zu bringen. Im vorliegenden Experiment wurde das Szenario geringer Liquidität mit einem Finanzierungshorizont von einem Jahr angesetzt, was für viele Biotechnologiefirmen der deutschen Branche ein realistischer Planungshorizont ist (Ernst \& Young, 2004). Dennoch könnte manchen Managern eine Allianzsuche unter diesen Umständen nicht sinnvoll erscheinen, wodurch auch Auswirkungen auf die in dieser Studie ermittelte Reihenfolge der anderen Entscheidungsparameter nicht ausgeschlossen werden können. Dies könnte mittels einer Adaptiven Conjoint-Analyse (Johnson, 1987) überprüft werden. Dieses Verfahren basiert auf trade-off Paarvergleichen von Entscheidungssituationen und ermöglicht eine „Aussortierung“ von nicht entscheidungsrelevanten Parametern bzw. von Parametern, für die ein Mindestniveau erwartet wird.

Schließlich ist es nicht selbstverständlich, dass die Ergebnisse dieser Arbeit auf andere Industrien übertragen werden können. Da junge Biotechnologiefirmen besonders kapitalintensiv sind (Herstatt, Müller, 2002), kann es sein, dass die teilnehmenden Manager in dieser Studie einen besonderen Wert auf Liquidität und das Finanzierungsumfeld legen und diese Faktoren in anderen Industrien, in denen Allianzen ebenso verbreitet sind (Hagedoorn, 1993), eine geringere Rolle spielen. Deshalb erscheint eine Überprüfung der Ergebnisse in anderen Branchen sinnvoll. 


\section{Anmerkungen}

* Danksagung: Der Autor dankt zwei anonymen Gutachtern für ihre hilfreichen Kommentare. Das Projekt wurde durch ein EXIST-HighTEPP-Stipendium des Bundesministeriums für Bildung und Forschung gefördert.

1 Zur Überprüfung der Validität der Zwei-Cluster-Lösung wurde auch eine Drei-Cluster-Lösung berechnet. Diese ergab allerdings zwei Cluster mit identischen Vorzeichen, die denen des Cluster 2 der Zwei-ClusterLösung entsprachen (siehe Tabelle 3). Daher wurde die Zwei-Cluster-Lösung aufgrund ihrer theoretischen Fundierung bevorzugt.

2 Die Verwendung alternativer Startpunkte (zufällige Werte der Koeffiziententabelle) ergab eine identische Clusterung.

\section{Literatur}

Acs Z. J., Audretsch D. B. (1989): Patents as a measure of innovative activity, in: Kyklos, Bd. 42, Heft 2, S. 171-180.

Alvarez S. A., Barney J. B. (2001): How entrepreneurial firms can benefit from alliances with large partners, in: The Academy of Management Executive, Bd. 15, Heft 1, S. 139-148.

Audretsch D. B., Feldman M. P. (2003): Small-firm strategic research partnerships: The case of biotechnology, in: Technology Analysis \& Strategic Management, Bd. 15, Heft 2, S. 273-288.

Barney J. (1991): Firm resources and sustained competitive advantage, in: Journal of Management, Bd. 17, Heft 1, S. 99-120.

Baum J. A., Oliver C. (1991): Institutional linkages and organizational mortality, in: Administrative Science Quarterly, Bd. 36, Heft 2, S. 187-218.

Brehmer A., Brehmer B. (1988): What have we learned about human judgment from thirty years of policy capturing? in: Brehmer, B., Joyce, C. R. B. (Hrsg.): Human Judgment: The SJT View, St. Louis, MO.

Brown T. R. (1972): A comparison of judgmental policy equations obtained from human judges under natural and contrived conditions, in: Mathematics Bioscience, Bd. 15, Heft 2, S. 205-230.

Brush C. G., Greene P. G., Hart M. M. (2001): From initial idea to unique advantage: The entrepreneurial challenge of constructing a resource base, in: Academy of Management Executive, Bd. 15, Heft 1, S. 64-78.

Chi T. (1994): Trading in strategic resources: Necessary conditions, transaction cost problems, and choice of exchange structure, in: Strategic Management Journal, Bd. 15, Heft 4, S. 271-290.

Coombs J. E., Mudambi R., Deeds D. L. (2006): An examination of the investments in U.S. biotechnology firms by foreign and domestic corporate partners, in: Journal of Business Venturing, Bd. 21, Heft 4, S. 405-428.

Das T. K., Teng B.-S. (2000): A resource-based theory of strategic alliances, in: Journal of Management, Bd. 26, Heft 1, S. 31-61.

DeCarolis D. M., Deeds D. L. (1999): The impact of stocks and flows of organizational knowledge on firm performance: An empirical investigation of the biotechnology industry, in: Strategic Management Journal, Bd. 20, Heft 10, S. 953-968.

Deeds D. L., DeCarolis D. M., Coombs J. E. (1997): The impact of firm-specific capabilities on the amount of capital raised in an initial public offering: Evidence from the biotechnology industry, in: Journal of Business Venturing, Bd. 12, Heft 1, S. 31-46.

Deeds D. L., DeCarolis D. M., Coombs J. E. (1998): Firm-specific resources and wealth creation in hightechnology ventures: Evidence from newly public biotechnology firms, in: Entrepreneurship Theory and Practice, Bd. 22, Heft 3, S. 55-73.

Deeds D. L., DeCarolis D. M., Coombs J. E. (1999): Dynamic capabilities and new product development in high technology ventures: An empirical analysis of new biotechnology firms, in: Journal of Business Venturing, Bd. 15, Heft 3, S. 211-229.

Deeds D. L., Hill C. W. L. (1996): Strategic alliances and the rate of new product development: An empirical study of entrepreneurial biotechnology firms, in: Journal of Business Venturing, Bd. 11, Heft 1, S. 41-55.

Deeds D. L., Hill C. W. L. (1998): An examination of opportunistic action within research alliances: Evidence from the biotechnology industry, in: Journal of Business Venturing, Bd. 14, Heft 2, S. 141-163.

Deeds D. L., Mang P. Y., Frandsen M. L. (2004): The influence of firms' and industries' legitimacy on the flow of capital into high-technology ventures, in: Strategic Organization, Bd. 2, Heft 1, S. 9-34. 
DiMasi J. A., Hansen R. W., Grabowski H. G. (2003): The price of innovation: New estimates of drug development costs, in: Journal of Health Economics, Bd. 22, Heft 2, S. 151-185.

Dollinger M. J. (1995): Entrepreneurship: Strategies and resources, Boston, MA.

Dollinger M. J., Golden P. A., Saxton T. (1997): The effect of reputation on the decision to joint venture, in: Strategic Management Journal, Bd. 18, Heft 2, S. 127-140.

Eisenhardt K. M., Schoonhoven C. B. (1996): Resource-based view of strategic alliance formation: Strategic and social effects in entrepreneurial firms, in: Organization Science, Bd. 7, Heft 2, S. 136-150.

Ernst \& Young. (2002): Beyond Borders - Global Biotechnology Report 2002, Cambridge, UK.

Ernst \& Young. (2004): Per Aspera ad Astra - Deutscher Biotechnologiereport 2004, Mannheim, Germany.

Ernst \& Young. (2005): Kräfte der Evolution - Deutscher Biotechnologiereport 2005, Mannheim, Germany.

Ernst H., Leptien C., Vitt J. (1999): Schlüsselerfinder in F\&E: Implikationen für das F\&E-Personalmanagement, in: Zeitschrift für Betriebswirtschaft, Bd. 69, Ergänzungsheft 1/1999, S. 91-118.

Ernst H., Omland N. (2003): Patentmanagement in jungen Technologieunternehmen, in: Zeitschrift für Betriebswirtschaft, Bd. 73, Ergänzungsheft 2/2003, S. 95-113.

Fildes R. A. (1990): Strategic challenges in commercializing biotechnology, in: California Management Review, Bd. 32, Heft 3, S. 63-72.

Gomes-Casseres B. (1996): The alliance revolution: The new shape of business rivalry, Cambridge, MA.

Gompers P. A., Lerner J. (1999): The Venture Capital Cycle, Cambridge, MA.

Green P. E. (2001): Foreword, in: Gustafsson, A., Herrmann, A., Huber, F. (Hrsg.): Conjoint measurement methods and applications, Berlin.

Gulati R. (1995): Does familiarity breed trust? The implications of repeated ties for contractual choice in alliances, in: Academy of Management Journal, Bd. 38, Heft 1, S. 85-112.

Hagedoorn J. (1993): Understanding the rationale of strategic technology partnering: Interorganizational modes of cooperation and sectoral differences, in: Strategic Management Journal, Bd. 14, Heft 5, S. 371385.

Hahn G., Shapiro S. (1966): A catalogue and computer program for the design and analysis of orthogonal symmetric and asymmetric fractional factorial designs, Schenectady, NY.

Hamel G., Doz Y. L., Prahalad C. K. (1989): Collaborate with your competitors - and win, in: Harvard Business Review, Bd. 67, Heft 1, S. 133-139.

Hammond K. R., Adelman L. (1976): Science, values, and human judgment, in: Science, Bd. 194, Heft 4263, S. 389-397.

Hays W. (1973): Statistics, New York.

Healy P. M., Kang S.-H., Palepu K. G. (1987): The effect of accounting procedure changes on CEO‘s cash salary and bonus compensation, in: Journal of Accounting \& Economics, Bd. 9, Heft 1, S. 7-34.

Herstatt C., Müller C. (2002): Management-Handbuch Biotechnologie. Strategien, Finanzen, Marketing, Recht, Stuttgart.

Hitt M. A., Ahlstrom D., Dacin M. T., Levitas E., Svobodina L. (2004): The institutional effects on strategic alliance partner selection in transition economies: China vs. Russia, in: Organization Science, Bd. 15, Heft 2, S. 173-185.

Johnson R. M. 1987. Adaptive Conjoint Analysis, Proceedings of the Sawtooth Software Conference on Perceptual Mapping, Conjoint Analysis, and Computer Interviewing. Sawtooth Software, Inc.: Ketchum, ID

Lerner J., Merges R. P. (1998): The control of technology alliances: An empirical analysis of the biotechnology industry, in: The Journal of Industrial Economics, Bd. 46, Heft 2, S. 125-156.

Lerner J., Shane H., Tsai A. (2003): Do equity financing cycles matter? Evidence from biotechnology alliances, in: Journal of Financial Economics, Bd. 67, Heft 3, S. 411-446.

Myers S. C., Majluf N. S. (1984): Corporate financing and investment decisions when firms have information that investors do not have, in: Journal of Financial Economics, Bd. 13, Heft 2, S. 187-221.

Pablo A. L. (1994): Determinants of acquisition integration level: A decision-making perspective, in: Academy of Management Journal, Bd. 37, Heft 4, S. 803-836.

Powell W. W., Koput K. W., Smith-Doerr L. (1996): Interorganizational collaboration and the locus of innovation: Networks of learning in biotechnology, in: Administrative Science Quaterly, Bd. 41, Heft 1, S. 116145.

Rothaermel F. T., Deeds D. L. (2004): Exploration and exploitation alliances in biotechnology: A system of new product development, in: Strategic Management Journal, Bd. 25, Heft 3, S. 201-221.

Schweizer L. (2005): Organizational integration of acquired biotechnology companies in pharmaceutical companies: The need for a hybrid approach, in: Academy of Management Journal, Bd. 48, Heft 6, S. p10511074. 
Shepherd D. A. (1999): Venture capitalists’ assessment of new venture survival, in: Management Science, Bd. 45, Heft 5, S. 621-632.

Shepherd D. A., Zacharakis A. (1997): Conjoint analysis: A window of opportunity for entrepreneurship research, in: Katz, J. (Hrsg.): Advances in Entrepreneurship, Firm Emergence and Growth, Greenwich, CT, S. 203-248.

Stewart T. R. (1988): Judgment analysis: Procedures, in: Brehmer, B., Joyce, C. R. B. (Hrsg.): Human Judgement: The SJT View, St. Louis, MO.

Wess L. (2002a): Germany’s shakeout, BioCentury, The Bernstein Report on BioBusiness: 21.10.2002.

Wess L. (2002b): Ingenium: Rethinking its approach, BioCentury, The Bernstein Report on BioBusiness: 08.12.2002.

Witt P., Rosenkranz S. (2002): Netzwerkbildung und Gründungserfolg, in: Zeitschrift für Betriebswirtschaft, Bd. 72, Ergänzungsheft 5/2002, S. 85-105.

Wittink D. R., Cattin P. (1989): Commercial use of conjoint analysis: An update, in: Journal of Marketing, Bd. 53, Heft 3, S. 91-96.

Zoltobrocki M. (2005): Profitabilität durch Neuausrichtung, in: Ernst \& Young (Hrsg.): Kräfte der Evolution Deutscher Biotechnologiereport 2005, Mannheim, Germany, S. 70.

zu Knyphausen D. (1993): Why are firms different? in: Die Betriebswirtschaft, Bd. 53, Heft 6, S. 771-792.

Zucker L. G., Darby M. R., Armstrong J. S. (2002): Commercializing knowledge: university science, knowledge capture, and firm performance in biotechnology, in: Management Science, Bd. 48, Heft 1, S. 138153. 


\section{Warum suchen Biotechnologieunternehmen strategische Allianzen?} Eine experimentelle Untersuchung

\section{Zusammenfassung}

In diesem Beitrag werden die Faktoren untersucht, die zur Allianzbildung junger Biotechnologieunternehmen beitragen. Aus einer ressourcenorientierten Perspektive wird argumentiert, dass die Entscheidung von Managern, Partnerunternehmen für neue Allianzen zu suchen, von der Ressourcensituation ihres Unternehmens und den Ressourcenanforderungen bzw. -akquisitionsmöglichkeiten der Umwelt abhängen. Eine Analyse von 1664 Allianzentscheidungen aus einem Conjoint-Experiment mit 52 Top-Managern der deutschen Biotechnologiebranche zeigt, dass fehlende finanzielle Ressourcen die Haupttriebkraft sind. Außerdem zeigt sich, dass für die meisten Manager fehlende, für manche Manager jedoch vorhandene Ressourcen eine stärkere Triebkraft der Allianzsuche darstellen. Diese Ergebnisse erweitern die wissenschaftliche Literatur über strategische Allianzen und haben Implikationen für die Managerpraxis.

\section{Why do biotechnology firms seek strategic alliances? An experimental study}

\section{Summary}

In this article we draw on a resource-based perspective to analyze the drivers for strategic alliance formation in the biotechnology industry. We state that the decisions of managers to seek out new alliance partners depend on the resource endowments of their ventures as well as the resource demands and opportunities the environment places for them. We use a conjoint experiment to collect data on 1664 alliance decisions nested within 52 top managers of German biopharmaceutical ventures. Our data show that missing financial resources are the strongest driver. Moreover, we find that whereas for most managers missing resources are drivers of alliance search, a minority of managers is more motivated by a strong resource position of their ventures. Our results extend the literature on strategic alliances and have implications for practitioners. 